\title{
Gravitation: Immediate Action at a Distance or Close Up Events?
}

Harmen H. Hollestelle, CommonRoom, Bandung/Sukabumi, West-Java, Indonesia harmhollestelle@yahoo.com

\begin{abstract}
Is immediate action at a distance, like gravitational attraction, imaginable using the contrary concept of close up, tactile, events? Tactile events, defined with the term 'tap-tapping' as a blind man does, described in a two-way spiritual interaction theory, are implemented in physics to understand gravitation from this respect. The quantum mechanical wave function reduction during measurements receives a new approach. Formulated is a new proof for Einstein's Equivalence Principle, extending it beyond locality, and a sketch of how tactile interaction could explain dark energy and an accelerated expansion of the universe. Dark energy and dark matter are examples starting from which to discuss properties of matter and space and gravitation as immediate tactile action rather than mediated action such as electromagnetism.
\end{abstract}

Keywords: Gravitation, General relativity, Immediate action at a distance, Spirituality, Tactile interaction, Dark energy, Dark matter, Cosmology, Wave function reduction

\section{1, Introduction: Tactile Interaction in Enactive Cognition and Physics}

\section{1, Gravitation and immediate action events}

Since Newton gravitation is described as an immediate action at a distance. A gravitational interaction is assumed to exist between objects with mass without there being a propagated intermediary, like there is for electromagnetism, i.e. photons. Maybe this is so because gravitational interaction is_symmetrical between all masses. The aim of this research is to investigate how this immediate character of gravitation can be understood by using a combination of measurement concepts from relativity theory. The description of observers and simultaneous events could in turn merit from the introduction of spirituality and spiritual initiative as already is ordinary in enactive cognition theory. The quantum mechanical wave function reduction as an event of observation receives a new meaning from the discussed examples.

\section{2, Cognition theory and inter-active spirituality}

Starting with concepts of enactive cognition theory, like a person's initiative and the cognitive boundary [Noë, 1], [Werner, 2], and considering these to be properties of objects and observers, I will apply those properties to describe measurements in physics. Sensorimotor knowhow and spiritual initiative combined are the central part of enactive cognition theory. I will define with the term tap-tapping a tactile inter-activity at a boundary, coming from both sides, like knocking at the door: you have to know how to knock and the door has to be there. Tap-tapping as a term and having a nearly similar meaning originally was introduced by Alva Noë, who is one of the pioneers of enactive cognition [Werner, 2]. One can imagine the relation between tap-tapping and palpating in medical practice. Spirituality in my description asks that enactive cognition is a two way process, not only involving a person's spiritual initiative, but also the actual presence and purpose of what is cognized, meaning inter-activity between person and object. Thus also tap-tapping in my definition implies a two-way interactivity. This will be defined more precisely below. It means that objects can posses a 'sort of' spiritual property, however only when they are given attention by a person. Two-way interactive spirituality I discussed in detail before in [Hollestelle, 3].

\section{3, Rigidity and fixed distance}


Local rigidity, familiar from Einstein's measuring rods [Einstein, 4], will be generalized towards extended spatial connectedness. Traditionally rigidity means that two points in space are connected at a fixed distance [Einstein, 5], (say: a 'plank' in Dutch). By proposing the existence of an extended spatially connected real object, like a rigid solid in contrast to for instance a gas cloud, I will make plausible one-time i.e. simultaneous knowledge by one observer of simultaneous events over non-infinitesimal distances. When the object as a whole is subjected to a push at one side towards a translation starting from rest, (say: 'klop' in Dutch), this one side of the object experiences an action and the other side is immediately, at the same time, causally connected. I will discuss this situation extensively with the help of Einstein's rigid measuring rods and simultaneous events in the reference frame.

\section{4, Causality between events depending on rigidity}

Causally connected events like this are an example of immediate interaction like I want to investigate here. Immediate action then, as it depends on the existence of rigidity as extended connectedness, will be argued to be a real and existing phenomenon. An extended spatially connected object does not contradict a quantum mechanical picture of a real rigid solid object with thermal fluctuations. And it does not contradict a maximum velocity of propagation, like the velocity of light in relativity theory. It concerns not propagation but instead simultaneous changes of motion during the start of the translation of the extended spatial connected object as a whole.

\section{5, Results: rewriting the equivalence principle, dark energy and dark matter}

It is the purpose of this research to implement concepts from inter-active tactile cognition and spirituality in measurement theory and to find how this can be helpful in understanding properties of matter. This is clarified with the example of a rigid object subject to translation and examples of quantum mechanical measurements in physics (star light observation, and the two slit experiment) in paragraphs 2 and 3. Spirituality and inter-active tactile cognition are discussed in more detail in paragraph 4 to 6. From there I am able to rewrite the equivalence principle, in paragraph 7. Finally dark energy and dark matter are discussed in the last paragraph 8 as natural examples of the application of tactile cognition. The equivalence principle can be understood to emerge from three theorems by Newton: one an addition property for infinitesimal distances, one a composition property for inverse square gravitational attraction and one a mean value property for gravitational attraction [Newton, 6], [Newton, 7]. Newton's first theorem was based on his calculus of fluxions that finds its origins already in classical Greek mathematics with the method of 'exhaustion' by Eudoxus and Archimedes and the method of the 'geometrical atom' by Democritus [Struik, 8].

\section{2, Gravitational Attraction and Immediate Action at a Distance, an Example}

\section{1, Classical extended objects in physics}

Many theories and applications in physics include a description of extended objects. For instance the 'radius' of the atom can be derived in early quantum mechanics following Bohr's atom model. Heisenberg defined the 'size' of the electron as the difference in the electron's position when two very fast particles hit it one after the other within a very short time interval [Beller, 9]. However classical particles are difficult to visualize in quantum mechanics in the wave description [Wichmann, 10], where one uses undividable wave packets, and probabilities are to preserve a link to measurements of undividable particles that cannot be point particles. Also, in relativity theory one speaks of necessarily classical objects and even human persons, like clocks and observers. However when considering the details in relativity theory of the description of movements for instance, it turns out that of the objects remain point particles in space having certain co-ordinates. Of course the fact that the mechanics of a system of particles can be simplified using the center of mass facilitated this development. Local space and locality are the concepts mostly used to maintain a link to experienced reality as it occurs to us and what should be part of measurement theory and 
practical reality in physics. In the case of relativity theory Einstein was well aware of the need of this link. In the case of quantum mechanics, as formulated by Bohr and Heisenberg in the 'Copenhagen interpretation', it is the reduction of the wave function that is interpreted as such [Beller, 9].

\section{2, Gravitation: equivalence principle and immediate action at a distance}

Einstein proposed to view gravitational attraction the same as acceleration, locally. This is the meaning of the equivalence principle. This interpretation is very successful towards an understanding of gravitation as a metric phenomenon. For many situations like with one object or person in a homogeneous gravitation field of a much larger object (say an observer on earth) gravitation for the observer can easily be understood by choosing a different metric for the observer's space. However this does not explain the immediate action at a distance character of gravitation. Action at a distance instead becomes a change of metric at a distance.

\section{3, An example of immediate causality at a distance during translation}

Immediate action at a distance can be better understood when exploring an example of the extended object mentioned already in the introduction. Imagine a non-infinitesimal rigid solid object that undergoes acceleration like a translation in one direction when starting from rest in a certain referential co-ordinate frame. Later I will consider the meaning of rigidity in more detail. It can be argued that both sides of the object in this direction experience acceleration at the same time and that the start of the acceleration can be experienced similarly, also at the same moment in time, by observers at both sides in the referential frame. For an observer placed at one side of the object, knowledge about the experience of an observer placed at the other side thus is immediate. When the translation is caused by a push at one side of the object the start of the movement of the other side is immediately caused by this event.

\section{4, Immediate action at a distance and interactive cognition}

The example is a special case of observers experiencing direct contact events with objects. I propose to discuss such contact events by considering enactive cognition theory to arrive at a two way interaction between persons and objects during observation. Later in paragraph 8 I will investigate how this can improve understanding of properties of dark energy and dark matter. Spirituality will be considered as essential for a description of interactions where human persons participate and for them free will is assumed, in contrast to determinism or mechanism. In particular, my enactivist description combined with a spirituality theory is essentially two way, two way between observer and object. The observer and the object are meeting. In reference [3] I describe in detail how spirituality can be introduced for both persons and objects. I will return to this later.

Since Newton gravitational mass is regarded to be the same as inertial mass in physics, and gravitation can only be an immediate inverse square attractive force [Dijksterhuis, 11]. Furthermore the equivalence principle introduced later by Einstein reads: A gravitational force locally is the same as a pseudo force experienced during acceleration. This implies that statically connected to a free falling observer locally is a Minkovskian metric [Einstein, 4]. Most of the terminology and concepts for his theory of gravitation are already defined by Einstein in his special theory of relativity. It is evident that an immediate force acting at a distance needs at least the concept of simultaneity. When introducing his theory of special relativity Einstein defined simultaneity by introducing the concepts of a stationary system, with rigid standards of measurement, immediate proximity of a place, local events, and a time definition by stationary clocks and synchronized remote clocks (all clocks in the stationary system) and a finite light velocity c. Also he introduced, to measure distances between for instance synchronized clocks in the stationary system, as rigid standard of measurement the local rigid measuring rod [Einstein, 4].

\section{5, Detailed example of a translation of a real rigid material object}


A finite translation of a rigid object can be described with a start moment, a certain time of continued movement with constant velocity and a stop moment. Imagine only the start of motion, an acceleration from rest in a stationary system, of the rigid material object $\mathrm{AB}$ with at both ends the positions $\mathrm{A}$ and $\mathrm{B}$. There is a change of position during some starter time lapse dt. Meant is a situation at rest say until time t0, after which the object starts to move away from its original position, for instance due to a temporary force, where the change in positions $\mathrm{dA}$ or $\mathrm{dB}$, is observed at time $\mathrm{td}$, just after $\mathrm{t} 0$, and $\mathrm{td}-\mathrm{t} 0=\mathrm{dt}$. The change in the positions and the time lapse will be chosen infinitesimal. In my notation a position is a single point in space and a place is a local region around a position.

Consider both ends A and B well inside the immediate proximity, local to the position, of A, during rest in the stationary system. When the motion starts both ends A and B move infinitesimally away from their original positions, however remain within immediate proximity of A's original position. Then all simultaneous events can be determined by one clock and one measuring rod. Of course now all causality is local and the start event at B clearly depends on the start event at A.

When B is outside immediate proximity of A their simultaneous time is the time of the stationary system in the reference frame (all notation following that from Einstein like above). Maybe it seems that an infinitesimal translation generalized towards a global translation is required to describe a real translation. However I assume that measurement of the infinitesimal motion, that is: infinitesimal translation, of A and B during the starter time lapse t0 to td is possible, and then no such global movement needs to be considered. A start-of-motion measurement, or in other words: start event experience, can be made and thus information transfer over a distance $\mathrm{AB}$ is true, where the local translation $\mathrm{dA}$ of $\mathrm{A}$ to $\mathrm{A}$ ' is the same as the local translation $\mathrm{dB}$ of $\mathrm{B}$ to $\mathrm{B}$ ', within first order approximation. This information transfer is independent of light signals, all that is needed are synchronized clocks measuring Atime and B-time: observation of the displacement of A and of B are occurring simultaneously at the same 'time of the stationary system', that is from t0 to td. Knowledge (by an observer at original place A) of displacement dA of end $\mathrm{A}$ gives immediate knowledge of the (similar) displacement $\mathrm{dB}$ of end $\mathrm{B}$ at a distance $\mathrm{AB}$, not counting the individual infinitesimal displacement differences that might be possible between ends $\mathrm{A}$ and $\mathrm{B}$. It is not so much the displacements $\mathrm{dA}$ or $\mathrm{dB}$, and $\mathrm{AB}$ or $\mathrm{A}$ 'B' as distances that are important, important is that the observation of the event 'start of displacement' at the time td at end A means a similar event is taking place at end B simultaneously at the same time td.

Distance A'B' is a distance to be recovered by 'operation (b)' defined by Einstein in [Einstein, 4]: i.e. the length of a moving object in the stationary system depends on the original stationary distance $\mathrm{AB}$ at time t0 just before the start of the motion and on the infinitesimal motion at the time of its observation $t d$, both $t 0$ and td being times of the stationary system. Since the motion in this case is a start of displacement, thus including an acceleration, its complete history from $t 0$ to td is needed to infer A'B' from AB. The object remains observed in the stationary system and thus simultaneous observations, however by different clocks, of its sides both before and during translation, is secured.

When the start of motion at time t0 is caused by a push at side A like assumed before, indeed start of motion at side $\mathrm{B}$ is immediately caused by the start of motion at side A. I depend for this result on the rigidity of the real material object. Rigidity should mean that its sides A and B are at a fixed distance. Even when somehow elasticity cannot be discarded I know that it is not the translation that counts, it is the start event itself that is crucial. Thus this still serves as an example of immediate action at a distance, and of immediate causality.

Quantum field theory assumes the existence of a vacuum cloud of virtual particles instead of an empty vacuum [Sakurai, 12]. Then a translation of AB could be damped by a virtual particles cloud. However this cannot reduce the validity of the above example situation of immediate causality. Again, it is not the translation, it is the start event itself that matters. Thus also this argument cannot refute the above example of immediate causality.

\section{Quantum Mechanical Wave Function Reduction in Terms of Tactile Events}

\section{1, Star Light Observation: the Cognitive Event}


To observe a light wave from a certain source, an observer will need to direct a sensor, sensitive in some range of electromagnetic radiation. Directing the sensor is what I term above the spiritual initiative. There is more to it than just rotating the sensor. The observer has knowhow to know where to look. Training can be experienced memory knowledge of former observations and/or feeling for how to perform the experiment, and includes theoretical knowledge. To direct the sensor is similar to exposure of a sensitivity or vulnerability towards existing light waves, to observe in an empathic and understanding way. Light from a source like a star will radiate from the star in all directions.

When the sensor finds the source a light wave is observed, as it appears as a photon during some time lapse, even while from the source's distance the light wave seems to be too faint and far away for photons to concentrate and occur within some possible sensor area. Observation in terms of cognition is a meeting event between a scarce wave packet and the experimenter through the sensor. This is the inter-active cognition event. The sensor stands in for the experimenter and is assumed to not exist alone by itself. For the sensor to capture, register, light, to confirm observation, it needs energy transfer like the capture of a photon. The light wave shell from the star reduces towards a photon, that in turn is absorbed [Wichmann, 10].

To understand the properties of the captured light one has to and can only compare with one's own properties, say in this case the sensor's properties. A light wave packet or photon's energy has to be compared with an energy level distance from the sensor as it is prepared. The levels so to speak should match and embrace the wave packet energy for similarity.

This experimental activity has spiritual meaning since it possesses a free choice element. Obviously an observer could try and measure in all other directions, and similarly all other directions are possible for light leaving any other star to reach the sensor, however he/she then would have to choose to direct the sensor in those other directions. As soon as the experiment starts, focused on photons in one specific direction, the QM wave function reduction takes place while simultaneously QM photons are found to appear in this one direction while received by the sensor. The event of observation, the capture of the photon by the sensor, is the cognitive event. It can be described by tap-tapping as tactile event in the following way. The observer constructs and orientates the sensor: in this way he/she enables himself to experience the encounter by reading the sensor when a photon meets the sensor and is captured. The human sense of tactile observation is so to speak forwarded towards the sensor surface. On the other hand the star radiates waves and with the waves its ability to transfer energy with the radiation, in all directions. The capture of a photon and its energy transfer is a rare moment of encounter of the observer and the star. Both seem to have somehow their own ways, that emerge during the encounter. The human observer has a freedom the star does not possess, a human can choose while a star has to do all possibilities. The reduction of the light wave function and the registering of the sensor encompass a two way meeting. I will call this meeting a tap-tapping encounter.

\section{2, The Two Slit Experiment}

During the well known two slit experiment light is emitted by one source towards a screen with two slits, with behind this first screen a second screen [Wichmann, 10]. As long as the distance between the slits is less compared to the light's wave length, an interference pattern can be observed, as cognitive event, at the second screen, after some time. The wave length of the light can encompass both slits. Thus this one wave is 'tap-tapping' its way through the situation towards the second screen while a pattern occurs, and the other way around, the experimenter is 'tap-taping' his/her way along the second screen to capture the light wave, directing a sensor at successive places at the screen. The light wave, in the tactile description, when passing the first screen and finding both the slits, goes through them both before arriving at the screen where it does or does not meet the sensor. Just as in the above example with a star light shell, the light source has to do it all. The experimenter chooses which places at the second screen to position the sensor, this can be few or many, or even all.

When however the experimenter observes at one of the slits this means he/she chooses any tap-tapping event to take place at this slit. The wave will reach both slits at the first screen. Then, like before, a photon observation at the place of the slit chosen by the experimenter is a cognitive event and means wave function reduction took place 
towards a captured photon at this place. At this event both the wave and the experimenter 'tap-tap' towards each other and meet at this slit. The use of the term tap-tapping here becomes visualized with the light wave ray finding its way just like the experimenter does illustrating the two way character of cognitive events. When the experimenter chooses to observe at both slits, an observation will occur depending on the wave reaching the first screen at both of the slits where in some time interval wave function reduction takes place and a photon can be captured at any of the two slits depending on probability. A practical time interval will relate to wave amplitude since wave energy and photon probability depend on it. Again here the light source reaches both slits while the experimenter can chose to measure at any slit in any time interval in turn.

\section{3, Tap-Tapping and the Reduction of the Wave Function}

To visualize what happens for instance when an experimenter tries to observe light, say when the source is a star, imagine the following situation. Star light, the light wave in QM description, is going in all directions at the same time. The idea is that the star as an object has to tap-tap all possibilities while the experimenter can choose one. The star does so by radiating, mediating, waves in all directions. This completely agrees with my description of spirituality: human persons have a free will and can choose according to their intention, whereas objects have spirituality only during the time they are given attention. Still, as objects cannot chose, they 'follow' the person's choice, just like wave function reduction at an oriented sensor. Only by including the observer as a spiritual person with initiative and knowhow, and objects as receiving temporary spirituality from the person, the complete situation of an experiment in physics can be understood and explained.

\section{Inter-active Cognition and Two Way Spirituality}

\section{1, Spirituality of Humans and Objects in inter-active Cognition}

I will depend on enactive cognition theory by Noë and Varela, Thompson and Rosh (denoted VTR), as their theory is described in [Noë, 1], [Block, 13], [Clark, 14] and [Hickerson, 15] on Noë, and [Basu, 16] and [Rangarajan, 17] on VTR, and [Werner, 2] on both. In enactive cognition theory perceptual experience includes the practical exercise of sensorimotor knowhow. Cognition is an activity: hence the term enactive. When cognition theory is a theory of enactivity, I will argue it is in need of support from a description of spirituality. I will start my investigation from there. In my definition spirituality is a human action of the inspired free will. To understand how spirituality 'works' in practice I then need to describe the interaction between person and person, and between persons and objects, the objects however having no free will. A detailed picture includes a notion of human free will and its 'response' from objects, giving arguments to define spirituality not only for persons, also for objects [Hollestelle, 3].

Spirituality defined this way does not align with determinism or mechanism. There is a sense of destiny like with 'true' co-operation, given the human freedom to decide for a new possibility at any moment. Human freedom exists and it exists in the choice to accept, or not to accept, at any moment, a gift (compare a calling or vocation) from_God that has many ways. My notion of freedom resembles someway how freedom occurs in Occasionalism, which is a medieval cosmology. The relation spirituality, causality, natural law and God is difficult to explain. One can argue that natural law is not constructed by humans, can only be discovered as property of objects [3]. This does not have to mean theories cannot change or vary. However it is beyond the scope of this research to discuss theology. Assuming spiritual co-operation between persons and objects including objects of nature, natural law receives its definiteness before inspired human action. To distinguish from enactive cognition I will use the terms inter-active cognition and two way spirituality.

Despite the emphasis on free choice a true co-operation is two-way: human freedom to choose depends among other things also on waiting for the other. In [Hollestelle, 18] I describe examples in the context of experiments in physics. Spirituality for objects, in my description, is only defined during the time an object is given attention by a person. The object maybe cannot give or return attention, however it can at least be like a helper for the spiritually active person. Being a helper gives the object spiritual quality. One may think here, for example, of works of art that can 
inspire human life. A 'spiritual' song can bring about change of life during singing. It is not just a person singing a text, both person and music seem to be meant for each other and vulnerable to each other.

The above notion of a complete two way spirituality between person and person, and person and object, gives meaning to a new definition of two way enactive cognition as inter-activity. To be able to understand cognition one has to further develop the concept of boundary: how is a person defined by his or her location and self, and how do person and object encounter (given we discuss cognition of a person experiencing an object, postponing discussing persons acting among themselves). This may explain also the details in situations of experiment in physics as already discussed in the above paragraph 3.

\section{2, Inter-active Cognition, Two Way Spirituality and the Location of the Cognitive Event}

To define inter-active cognition again I start from the concepts introduced by Varela, Thompson and Rosh (VTR), and Noë. VTR assume: 'when we enlarge the task domains from artificial microworlds to the world at large, it is not clear that we can even specify what is to count as an object independent of the type of action that is being performed. The individuation of objects, properties, and events appears to vary according to the task at hand.' (Varela et al, 1991), [Werner, 2].

And: 'Cognition is not the representation of a pregiven world by a pregiven mind but is rather the enactment of the world and a mind on the basis of a history of the variety of actions that a being in the world performs' (Varela et al, 1991), [Werner, 2].

Enactive cognition as defined by VTR depends more on cognitive events and less on the human self. I will not follow them in this respect. The description by Noë seems to me to be more real since it allows a person a clear self and personal initiative.

For Noë, sensorimotor knowhow and understanding are essential in cognition: 'Perceiving is an activity of exploring the environment drawing on an understanding of the ways in which one's movements affect one's sensory relations to things.' [Noë, 1]

The enactive description completes two main cognitive theories: sense data theory and naïve realism. Sense data theorists argue that what you perceive are mental sense data strictly connected to the object perceived, while you go beyond this when complete these sense data towards a complete integrated mental image. Naïve realists argue that what we perceive is the real object with its properties independent of how it appears, for instance from an angle, as a profile. Sense data theorists fail to notice that mental sense data alone cannot be a faithful description of perception when what you perceive is a factual object. However naïve realists do not value the profile of an object enough as being an appearance [Noë, 1]. Noë: "we see how things are and we see how things are from here." "The solution to the problem of perceptual presence consists in recognizing that for a perceiver with the requisite understanding, seeing how things look can be an encounter with how things are. Just as holding your hand can be a way of holding you..."

When perceiving is an understanding encounter with things, you expect that there is a certain equivalence between perceiver and perceived. A person encounters an object at a boundary that is defined by their both spirituality where the person's spirituality defines a direction towards the other. A simple example is tactile perceiving like holding your hand.

Tactile perceiving like this, with a movement towards the other as a free will initiative, is newly performed at least at the first encounter and can be continued, just like knocking on the door. Thus defined is a boundary, the surface where to knock at the door and the surface of the hand knocking. The boundary surface defines and marks the location of the event of perceiving. Because perceiving is a movement, an activity, the location usually embraces more space than just a single point in space. That is why one speaks of the location of the cognitive event.

I define tap-tapping as a tactile movement towards the other which means exposing a sensitivity or vulnerability of yourself towards the existence of the other. Before perceiving, without yet perceiving the other, is just the movement of the exposure, as a search or an experiment preparing to find a boundary, it is like preparing yourself towards more 
understanding. Sometimes tap-tapping means an activity that asks to be continued, needs some time unto confirmation. Then it is like following the other in cognition. This seems to be so when a person and the boundary of the self ask stability [Werner, 2]. Tap-tapping has a special ring because it describes perfectly how a blind man finds his way. The term tap-tapping itself originates from Noë, according to Werner in [Werner, 2]. However Werner continues: 'On the contrary,...., Nö̈ wants to convince us, that all perceiving is like a blind person's tap-tapping. This illustration,......., is supposed to convey the message that perception establishes a closer tie between the cognizer and the environment than the fixation on vision should suggest. ' Clearly Werner does not agree with Noë. He suggests that tap-tapping brings no real connection to the self and resembles fumbling. To me tactile cognition seems to be a complete description when cognition is cognition at a boundary of the self. There, at the boundary, it is immediate and direct and true. However this is not the place to discuss the self further. I understand tap-tapping as a, tactile, movement and exposure. As such it has a wide scope and can even be part of an object's movement.

\section{5: Measuring and Comparing: 'Tract' and 'Embrace'}

With a surface, of a person or an object, one may mean that part of the object that is sensible to touching by another object without changing the object's shape [Hollestelle, 3]. To understand the properties of the perceived object one can compare with one's own properties. Just embrace the object to understand its overall measure compared to your own. From there properties can be translated to meaning like greater than, smaller than, equal with, for the values of the properties one has in common. A constraint is that embracing can only be local. Non-locality would spoil the understanding and feeling, in one piece, of an embrace. An embrace is intimately connected to a person's soul and live body.

Measuring a property then is comparing, essentially. The person's properties define the object's properties as far as they can be compared by embracing. A person's spiritual property, intention, introduces a not similar however equivalent property for the object, like purpose is, that can be compared with intention. Since any embrace adapts part of its ends from the embraced object, the relation between person and embraced object is inter-active and equivalent.

To arrive at a more general definition of 'embrace' in geometric measuring, including locality as above, recall Einstein's introduction of the rigid measuring rod locally as a basis for the definition of measuring distances and of the metric [Einstein, 4]. A measuring rod allows for distances to be added. To measure non-local distances one may start from adding infinitesimal distances, each measured by local measuring rods. Adding infinitesimal distances is a well known method in mechanics and calculus that can be considered to originate from Newton, [Newton, 6], [Goldstein, 19], [Arnold, 20]. One measuring rod then is equivalent to a standard tangent along a direction, locally. Along a direction two locally close-by positions in space are sufficient to create a standard tangent, in one piece, that is local and connected and a measure for the distance between the two positions. This is similar to a 'tract' as the defining part of a physical measuring rod, defined by Einstein [6]. The interpretation of standard tangents along infinitesimally distant places in space along a direction, does not pose a problem here because the limit towards one point in space is not needed, indeed one is moving the other way towards finite separation. This is in contrast to the standard definition of derivatives where this limit is required [Goldstein, 19].

A rigid measuring rod should be measuring always the same local distance, independent of local translation, assuming it to be locally rigid. Physically the measuring rod should be a practically-rigid solid. Of course a practically-rigid solid is not completely equivalent to an invariant real distance or a geometrical distance since it is subject to changes depending on physical entities like temperature [Einstein, 21]. Einstein gives Poincaré full credit for emphasizing the distinction between properties of physical practically realistic objects and geometry. However changes of properties like volume according to temperature could in principle be detected and recalibrated. For this purpose, one may compare properties of solids at different temperatures. Tracts of measuring rods like recalibrated rigid solids, differing from practically-rigid solids, could be, in principle, equivalent to invariant real distances and to geometrical distances. 


\section{Comparing and Counting Properties}

When considering a local place in Minkovskian space one assumes space-like local invariant real distances in three independent directions, and in every direction a rigid measuring rod can measure these real distances. With local place I mean a part of space where the distances between positions in it can be measured with one measuring rod in one time, just following Einstein's description. Now I will try to describe how numbers and counting are involved in measuring.

In [Hollestelle, 3] I discuss comparing and counting as follows: "Counting may have several different appearances (Murawski, 2010): counting may mean counting again and again the same event (say 'heartbeat'). Or it may mean counting along a scheme, resulting in the number 'one' as singularity, 'two' as spin, etc. Then there is counting like I want to discuss it here, namely counting as comparing, due to Euklid (Euklid, transl. C. Thaer, 1991): defining as 'one' a certain measure that is the property of a certain object and then comparing the next object and a related measure with it. In this last instance counting is comparing 'one' with the notion: another 'one'. This other 'one' can be larger than or smaller than the original 'one' or without difference. Generally one is interested in what then the next number is starting with a certain given original number. The next number follows on the original number, ideally with only a 'one' as difference. It is the possibility of noticing change and difference that makes comparing possible and makes the existence of successive numbers possible. In this description numbers are ontologically dependent, a proof of ontological dependence of numbers is given in (Schwartzkopff, 2011). Thus the reciprocity between the properties of objects, being themselves ontologically dependent on the objects, and their description in terms of numbers is consistent." (references in the article)

And: 'There is reciprocity between the properties of an object and its description in terms of numbers. Extension of an object can be described with the numbers that relate to its spatial shape. Concepts like 'belong to' and 'being connected to' find their counterpart in these numbers. Naively the shape of an object is reflected in the symmetries and similarities between the describing numbers. What is 'inside' the object is reflected for each direction by an interval, a series of numbers that all are below or equal to a certain value that represents the surface.' Again, with surface I mean that part of the object that is sensible to touching by another object without changing the object's shape.

Measuring then is continuing until. When relating to a physical reality, like a part of space or an extended object in space, the numbers that are the result of measuring, of measurements, are properties of this reality. Measurement of extension is continuing in one direction, starting from some chosen point at the object, until one stops continuing at the surface. From here it is only a small step towards the measuring rod. A measuring rod ideally would have the number 'one' as property, as its value for distance in its direction. To count then is adding and counting measuring rods and continuing until some surface or point in space is reached relevant to the to be measured distance. Of course only certain properties, like distances, can be measured directly in this way. Other properties can be measured when a scale is devised that translates a property towards a distance, like for instance a weight scale or a thermometer. When like in this article spirituality and intention are considered a person's property, this can only be measured indirectly, if at all. Any change, difference, occurring in an object that is given attention in a spiritual way, might give a misinterpretation. The object's properties, like differences before and after receiving attention, together can only be an mere indication of the object's purpose.

\section{Gravitational Attraction, Geometry, and a New Proof of Einstein's Equivalence Principle from three theorems by Newton}

\section{1, Newton's composition requirement for inverse square attraction}

Let us now visualize similarly a sphere with as surface a local boundary defined by the reach of a local standard measuring rod, assuming that locally all directions are measured equally. This latter requirement could be relieved 
later. This means that the sphere has a radius similar to the distance indicated by the measuring rod. The sphere surface is assumed to have uniform mass density, and has a_spherical symmetry. The first of the theorems by Newton I will use is that for any sphere, as a solid object, when composed of parts of matter, only with an inverse square attraction by the parts, a similar inverse square attraction holds outside the sphere, proportional to the total quantity of matter belonging to the sphere as a whole [Newton, 7], (pg 303 commentary] (say: composition requirement $\mathrm{R} 1$ ).

\section{2, Addition requirement from Newton's calculus for the standard measuring rod}

The second theorem of Newton needed is from his calculus: addition of distances measured from the middle of the sphere every time with one single infinitesimal and local measuring rod applied successively will increase linearly the sphere radius also to non-local distances [Newton, 6] (say: addition requirement R2). This process is the reverse of what is often called the 'usual limiting process' to define derivatives [Goldstein, 19]. In our case the lower limit is not infinitely small but equal to the standard measuring rod, which is the start of our reverse process to a non-local sphere surface.

According to General Relativity the actual length of a measuring rod will depend on the distance along the radius to the center of the sphere if for instance a point-like mass is assumed at the center. In that case the limit of the length of the measuring rod towards the sphere center at zero radius would diverge [Einstein, 21]. However this does not pose a problem here, since I do not assume point-like masses, only mass densities for a non-zero volume. The actual length of measuring rods is not an issue in the above reversed limiting process.

According to the above step by step addition process, a metric, when it measures a distance that can with each step be equated with a certain sphere radius, is nothing else than counting a measurement of separation in terms of standard measuring rods, counting a distance between two: the sphere middle and the boundary surface. Then, a geometry is always related to distance inside its definition space, here around the sphere middle inside the sphere surface. This would mean also, a geometry can only be defined consistently in a finite geometrical space. One always needs two sides to measure a geometrical or real distance. Indeed the above addition of real distances starting with a sphere with a radius similar to one measuring rod, cannot be reversed starting from infinity. And, the initial gravitational attraction at infinity would be zero.

\section{3, Newton's mean value requirement for inverse square attraction}

Inside the sphere surface, the homogeneous inverse square attraction is cancelled out, when adding together all attraction from the surface mass density as a whole [Newton, 7], (pg. 303 proposition LXX] (say : mean value requirement R3). Outside the sphere surface, inverse square attraction can be continued for any real distance and proportional to the sphere's total matter (R1). Attraction for infinite distances is not well defined since infinite radius or distance cannot be reached with the above process.

Now follows some terminology. Of the sphere surface, with the point $B$ in one radius direction from the sphere center $\mathrm{A}$, a sphere part like a local dot $\mathrm{C}$, in the same direction as $\mathrm{B}$, defines the sphere radius distance to be measured from the sphere middle A, while the gravitational attraction of the surface depends inverse squarely on the (to be measured) distance AD for an object D well outside the sphere. We choose A in such a way that A and B and $\operatorname{dot} \mathrm{C}$ and $\mathrm{D}$ are aligned. Indeed in the above definition of measurements the addition of measuring rods can be continued until dotC is reached and a metric is defined inside the sphere surface through $\operatorname{dot} C$

Inside the sphere surface (or even inside a solid sphere) the attraction is zero, however a metric is recovered, outside the sphere surface or solid sphere, the attraction is recovered however the metric is not defined. The sphere surface is assumed to be the outer limit of adding distances by applying measuring rods until dotC is reached from A. One notices that to recover the complete metric one has to enlarge, by applying more measuring rods, the sphere radius from within until dotC is further away from A than B is and reaches D. However to recover the attraction one has to 
reduce the sphere radius again from outside, say from $\mathrm{D}$, by reducing the number of applied measuring rods, until $\operatorname{dot} C$ again is at the same distance from A as B or less. One cannot recover both metric and attraction at the same time. By measurements one after the other one finds distances BD and dotCD and gravitational attraction $\mathrm{BD}$ as complimentary.

\section{4, Increasing a sphere surface mass density around the origin infinitesimally towards a place of attraction defines the relation between metric and inverse square attraction}

One may increase step by step the radius of the sphere surface along with $\operatorname{dot} C$ until $\operatorname{dot} C$ reaches the object $\mathrm{D}$. The sphere surface will appear approximately flat when approaching D closely. Object D when at close distance, say one step away, of dotC, will experience an attraction relative to its distance inverse squared measured from $\mathrm{A}$. This distance can be measured by 'looking back' in the step by step process after dotC reaches D. However, measured from the position of $\operatorname{dot} C$ one step before the arrival at $\mathrm{D}$ when the sphere surface at dotC appears flat to $\mathrm{D}$, the gravitational energy difference will turn out to be relative to the measured distance dotCD itself at that step. This is similar as it approximately is for instance for an observer on earth [Arnold, 20].

Thus the difference in metric distance found by continuing in one step with dotC towards $\mathrm{D}$, is proportional to the difference in gravitational energy for object $\mathrm{D}$ when the sphere surface and $\operatorname{dot} \mathrm{C}$ are still one step away. The process is that dotC and $\mathrm{D}$ make steps one by one alternately in a series where firstly after one step by $\mathrm{D}$ the difference in gravitation energy can be measured when dotC is still one step behind on $\mathrm{D}$ and dotC 'looks forwards' to $\mathrm{D}$, and secondly after a similar step by dotC the difference in metric distance can be measured while dotC catches up with $\mathrm{D}$ and together they 'look back' on their both one step earlier, and they continue like this until the final position of D is reached. With each step of $\operatorname{dotC}$ and the sphere surface requirement $\mathrm{R} 1$ is applied. In the process object D moves from B to its final position as it should, always on or just one step further than the sphere surface, creating its gravitational potential energy.

In this way one can generalize towards proportionality between metric and gravitational potential energy between the sphere surface encompassing $\mathrm{B}$, and the object $\mathrm{D}$ in all situations where the position of $\mathrm{A}$ is far away enough from B and D. In this way one recovers a new proof and understanding of Einstein's equivalence principle, in its general form extended beyond locality, using only R1, R2, and R3.

\section{Discussion: Dark Energy, Dark Matter and Close Up Events}

Close up, tactile events can be described with what above is defined as tap-tapping when spirituality is taken serious as a property of both persons and objects. Immediate action at a distance and close up events, and tactile events and QM observation are new combinations that need introduction in physics from scratch. Descriptions of both dark energy and dark matter can profit from this approach. Indeed tactile events do not depend on visual cognition, just like the property 'dark' suggests. Experimental evidence made the existence of dark energy and dark matter plausible because somehow energy and matter where missing, while they remained un-noticed by sight. Below I describe how close up events could be a good approach for both. The discussion why energy and matter should be dark or luminous is the most difficult to answer.

\section{1, Dark Energy}

As is well known the universe background radiation suggests that the universe is expanding in a accelerated way. This expansion is explained by adding a cosmological constant $\Lambda$ to the gravitational field equation. This addition implies the existence of a constant (dark) energy in all space or equivalently a dark energy density intrinsic to space itself. It is possible to derive within the quantum mechanical close up event description of paragraph 3. applied to the universe as a whole, an accelerated expansion just as well. For this I need to make some bold assumptions. This can only be a sketch of the real situation. 
The three laws of Newton, as basic to all physics, are summarized by Nijboer [Nijboer, 23], as: 1; an_object in a socalled inertial system experiences no external forces, 2; external force is equal to the derivative of momentum, heavy mass is similar to_gravitational mass and being in a gravitational field is equivalent to being in a accelerating system, 3; action is re-action.

This means according to 1; an object in a non-inertial system experiences an external force and thus that acceleration needs an external force. Because of the third part of 2; there seems to be some self-evidence: what should acceleration mean when there is no external force? Of course one has to read this part in combination with the second part of 2;. This thus also means mass/matter exists as property of an object in a accelerated system just as well as in a gravitational field.

The second law of Newton gives a preparation for how I will continue. Consider now a spherically symmetric finite universe with an outer shell as outer limit. This is possible since a metric measuring finite distances and thus spherical symmetry can be defined while staying within the finite limit of the universe, the metric domain. A infinite universe would not allow for the assumption of spherical symmetry. Let this symmetrical universe be host of mass/matter and let an energy density that is not necessarily symmetrical reside at the outer limit shell, thus a potential gravitational energy domain. The outer limit domain hosts a gravitation energy density rather than localized mass/matter objects since in this domain distances cannot be measured and have no meaning. It is out of reach of a metric because it is outside the finite inner metric domain, while a metric can only exist between two positions, and needs a finite space for it to measure distances between these two. The inner domain is defined as that part of the universe where distances do have meaning. The two domains thus have different character and have to be separate from each other. Consider this as a simple model for the universe.

Without further defining the boundary near the outer limit one can argue as follows. Assume an observer at the universe center. A measurement in a certain direction of potential gravitational energy will mean according to quantum mechanics that at a certain moment in that direction, all the energy of the outer limit domain will be experienced within an area, say E, in that direction at the outer limit domain. Thus, the outer limit being an energy density domain, an attraction outwards in the direction towards the boundary, towards E, will be measured. Gravitational attraction will be experienced by the observer inside towards outside. This will be so for any measurement in whatever direction in the inner metric universe domain, ruled by probability, when the energy at E, and the center of mass of the universe gravitate, pull at each other. The universe is accelerating in all directions outwards. This situation as an observation at the center of mass is similar to the quantum mechanical situation of the observation of a star light wave shell at a large distance such as at earth, as described before in paragraph 3 . Notice that now the area E, the place of the energy at the outer limit after reduction, is at a distance away from the observer at the universe center. The observer experiences the attraction as an immediate action at a distance. A device for measuring this attraction in one direction is imaginable, based on the fact that massive objects tend to start and accelerate due to gravitational attraction. It would then need two points at fixed distance and local to the observer to experience this behavior. A measurement like this would bring the place of experience in direct contact with the observer, immediate action at a distance would then become a close up event. (However this would mean a close observation of something else's gravitation.)

We may assume accelerated expansion instead of attraction according to the equivalence principle, along one direction. A finite universe will always have some center. Combining opposite directions the observer will experience opposite attractions according to probability. In the inner domain cancelling out of attractions mathematically does not mean that 'nothing happens' there. At its boundary and at each point in the inner domain there will exist a constant negative pressure (as derived below), which is equivalent to a constant energy per volume. When in a cosmological image the observer is discarded as having mass and the observer chooses to measure in all directions, without specific directions, the inner universe as a whole will experience accelerated expansion and can be considered to be tap-tapping at the outer limit as a whole instead of only at E. Because the reduction of energy density towards $\mathrm{E}$ is a QM probabilistic phenomenon, a Brownian motion of the center of mass of the inner universe is expected. The area $\mathrm{E}$ as the focus of tap-tapping will be 'traveling' around the outer surface. This does not mean it 
will cover the whole surface in time, only arrive infinitesimally near every point on it. This will give the opportunity to the realistic total surface area as the sum of all $\mathrm{E}$ in time to be smaller than the whole surface. Thus the inner pressure and the measure for the amount of dark energy will be higher than expected with a similar factor. A Comparison with indecomposable continua can be made when $\mathrm{E}$ follows three or more open domains on the surface each as series. In the case of three open domains, say like digging three canals in the example of the Lakes of Wada, the multiplication factor is 10/3 [Yoneyama, 24], [Hocking and Young, 25]. Such factors could explain the high amount of dark energy found to be present in the universe.

The distance to reach the outer domain from the center of the inner universe can be measured along a radius towards the outer limit. The potential energy, then, is proportional to the fixed mass inside the universe, the fixed mass density at the outer limit, a factor according to the inverse distance law for potential gravitational energy, and a factor proportional to radius squared according to the reduction towards $E$ of the energy density of the outer limit surface as a whole. Thus the potential energy is proportional to the universe radius, and the force at the distance of the radius, i.e. the outer surface, will be proportional to radius squared. At each point inside the inner domain opposite forces from the surface will cancel causing a constant negative pressure at the surface and thus at each inner point.

\section{2, Dark matter}

There has been a discussion with a long history concerning the matter content of galaxies during their formation period. A large percentage of matter in galaxies is called dark matter because it was only noticed by its gravitational influence on rotation curves. Dark matter hinders proto-star gas from star forming [Tinsley, 26] [Van Albada, 27]. Although the relation between dark matter and luminous matter with rotation curves has already been resolved [Navarro, 28] it can be a good example of how dark matter interacts.

It seems when dark matter consists of particles in high concentration through the proto-star gas it hinders star formation, just as according to Tinsley. Thus in turn the galaxy will be slow to acquire stars. At low concentration the star forming process will be less hindered and the galaxy will acquire stars earlier. The first type of galaxy will tend to have a less expressive center, the second type of galaxy a more expressive center. The process how dark matter can hinder star formation can be understood when one considers close up events to be important in its interactions. It is screening where dark matter particles are in the way of luminous gas particles.

A process that is interesting in this respect is the Higgs field mechanism. Higgs fields or Higgs particles are a possible candidate for dark matter [Hollestelle, 29]. The Higgs field does not react with photons and photons do not acquire mass, thus the Higgs field is dark. For a particle to acquire mass through the Higgs field mechanism, it has to be continuously interacting with the unstable Higgs field to confirm its mass. During this process the Higgs field will stabilize towards its lowest energy state. For mass acquiring particles it is like swimming against the stream. This is very much like a close up situation.

For stars to form out of a proto-star gas there is needed mass to gravitate. When more dark matter, say with a still unstable Higgs field, is present this means less mass is acquired and the galaxy will be slow to accumulate. When less dark matter is present, meaning an already stabilized lowest energy Higgs field, more mass will have been acquired and the galaxy will be fast to accumulate. In the second case the unstable Higgs field is more heavily burdened with the production of mass and Higgs particles compared to the first case. Dark matter then seems to correlate with the full unstable Higgs field rather than with Higgs particles.

There is a relation with the former paragraph on dark energy. Within a simple model universe dark energy emerges as a constant energy density inherent to space, like negative pressure. If dark matter correlates with the Higgs field a similar picture emerges. (More) dark matter in this picture means less mass produced through the Higgs mechanism. Thus dark matter as Higgs field is the opposite of baryon mass/matter content in space and measures free space. The idea is that whereas baryon mass/matter between itself gravitates in the ordinary sense and exerts positive pressure in this way, dark matter will gravitate in the opposite sense and exert negative pressure.

In that case one understands the Higgs mechanism as follows: dark matter/Higgs field negative pressure, directed towards outside everywhere in space, is interpreted as positive pressure, compression, everywhere in space towards 
baryon matter that is slowed down transforming the pressure energy into mass. Of course this process has to be repeated continuously to not lose the positive pressure and lose mass. This process may give an idea of how action at a distance is possible for gravitation: when mass and gravitation is the result of pressure from dark matter Higgs fields everywhere in the universe's space, and the universe is assumed to be finite like the model universe above, then since pressure is experienced similarly in all places in the space of this universe, gravitation will be experienced similarly everywhere too. This means that, just like pressure, the action of gravitation is experienced as close up event just as well as it is an action at a distance. This relation between close up events and gravitation deserves further study.

\section{References:}

[1] Noë, A.: Précis of Action in Perception, Philosophy and Phenomenological Research LXXVI, nr. 3, (2008)

[2] Werner, K.: Enactment and Construction of the Cognitive Niche, Synthese, (March 2018)

[3] Hollestelle, H.: Comparing the Incomparable, The Int. Jrnl. of Humanities and Social Studies, 5:4 (2017)

[4] Einstein, A.: On the Electrodynamics of Moving Bodies in Lorentz, H., Einstein, A., Minkovski, H. and H. Weyl: The Principle of Relativity, translated by Perrett, W. and G. Jeffery, Dover, (1952), reprint of the original publication, Methuen and Company, 1923

[5] Einstein, A.: Geometry and Experience, Expanded Version of a Lecture before the Prussian Academy of Sciences in Berlin on January 27 ${ }^{\text {th }}$, 1921, The Collected Papers of Albert Einstein, Princeton University Press, (2002), facsimile of the original publication, Springer Verlag, 1921

[6] Newton, I.: Quadrature of Curves, Editor D. Wilkins, (2014), Reprint of the English translation (by J. Harris, 1710) of Quadratura Curvarum, 1676

[7] Newton, I.: Mathematical Principles of Natural Philosophy, Editor Dawsons of Pall Mall, (1968), facsimile of the English edition, Andrew Motte, 1729

[8] Struik, D.: A Concise History of Mathematics, Dover, (1987)

[9] Beller, M.: Quantum Dialogue, The University of Chicago Press, (1999)

[10] Wichmann, E.: Quantum Physics, Berkeley Physics Course Vol. 4, McGraw-Hill, (1971)

[11] Dijksterhuis, E.: De Mechanisering van het Wereldbeeld (The Mechanisation of the World Image), Meulenhoff, (2000)

[12] Sakurai, J.: Advanced Quantum Mechanics, Addison-Wesley, (1978)

[13] Block, N.: Action in Perception by Alva Noë, The Jrnl. of Philosophy, (January 2005)

[14] Clark, A.: Vision as Dance? Three Challenges for Sensorimotor Contingency Theory, Univ. of Edinburgh, Association for the Study of Consciousness, (2006), http://www.hdl.handle.net/1842/1444

[15] Hickerson, R.: Knowing how to Possibly Act: Alva Noë’s Action in Perception, Philosophical Psychology, 20(4), (2007)

[16] Basu, M.: Book Review: A. Noë: The Embodied Mind, Complicity 1:1, (2004)

[17] Rangarajan, A.: Book Review: Varela, F. Thomson, E., and E. Rosch: The Embodied Mind, (1997), Univ. of Florida, https://www.researchgate.net/publication/2780884

[18] Hollestelle, H.: Do You Know Where to Look, (2010), arXiv.org:1011.6300

[19] Goldstein, H.: Classical Mechanics, Addison-Wesley, (1950)

[20] Arnold, V.: Mathematical Methods of Classical Mechanics, Springer Verlag, (1989)

[21] Einstein, A.: The Foundation of the General Theory of Relativity in Lorentz, H., Einstein, A., Minkovski, H. and H. Weyl: The Principle of Relativity, translated by Perrett, W. and G. Jeffery, Dover, (1952), reprint of the original publication, Methuen and Company, 1923

[23] Nijboer, B.; Voortgezette Klassieke Mechanica (Advanced Classical Mechanics), Utrecht University Graduate Course, (1974)

[24] Yoneyama, K.: Theory of Continuous Sets of Points, Tohoku Math. Jrnl. 12, (1917)

[25] Hocking, J., and G. Young: Topology, Addison-Wesley, (1961) 
[26] Tinsley, B.: Correlation of the Dark Mass in Galaxies with Hubble Type, Mon. Not. R. astr. Soc. 194:63, (1981)

[27] Van Albada, T. and R. Sancisi: Dark Matter in Spiral Galaxies, Phil. Trans. R. Soc. Lond. A 320:447, (1986)

[28] Navarro, J.: Cosmological Constraints from Rotation Curves of Disk Galaxies, (1996), arXiv.org:astro$\mathrm{ph} / 9610188$

[29] Hollestelle, H.: A New Virial Equilibrium for Galaxy Clusters Forming Finite Alignments, The Int. Jrnl. of Science and Technoledge, 5:10, (2017) 